DOI: $10.1134 / \mathrm{S} 1019331614030010$

\title{
What Is Wrong with Hirsch?
}

The Hirsch index is universally known. Attitudes to it vary: from favor (primarily on the part of those whose $h$-index is high) to scorn (on the part of those with a low $h$-index). Its drawbacks and advantages are widely and hotly debated at all levels - from junior researchers to the Minister of Education and Science. The majority of RAS members have a Hirsch index of 10 to 30, i.e., at the level of many of Nobel laureates before they won their Nobel prizes. However, there is a group of outstanding scientists whose $h$-index is $40-60$ and higher: I.M. Khalatnikov, A.A. Starobinskii, V.E. Fortov, V.E. Zakharov, L.D. Faddeev, Zh.I. Alferov, S.P. Novikov, V.P. Skulachev, and A.R. Khokhlov. Yet their scientific leadership is recognized without any Hirsch index. (Anticipating the typically Russian question "And what about you? What is your $h$-index?," let me answer: it is modest, 36 .)

Now let us ask ourselves another question: what Hirsch index would Einstein have had when he published his theory (imagine that this index existed at that time)? Right: zero or next to zero. Remember that only journalists wrote about his theory and used to call it extreme madness, a crazy trick, a shameful baby of a feebleminded brain, pseudoscience, squint-eyed physics, and so on. Even comments of great minds were far from being complimentary. The legendary $\mathrm{O}$. Heaviside understood Einstein's theory at once but found it senseless. The famous physicist A. Michelson called it incompatible with common sense. N. Tesla ridiculed Einstein's ideas and considered his formula $E=m c^{2}$ an illusion. The great E. Mach ignored this theory. T. See, the then dean of the Department of Astronomy at the University of Chicago, wrote that Einstein was a mere bungler and his theory was a fallacy and sophistry. Yet the most disgusting comment belonged to the vehement anti-Semite P. Lenard, a German physicist with fascist views, who considered Einstein's theory "Jewish science." Einstein received his Nobel Prize not for the theory of relativity, his greatest achievement, but for the physics of the photoelectric effect.

The great theory of Maxwell began an article that he had written in his student years at the University of Cambridge. Of course, no one read this article. Recognition made a very tardy appearance. The famous Lord Kelvin considered the brilliant discovery of X-rays, which have been serving humankind for more than a century, a mystification. B.P. Belousov (1893-1970), the discoverer of amazingly beautiful oscillation modes of chemical reac- tions, which dominated all significant biological processes, such as thinking, heart and muscle performance, and many others, was never recognized in his lifetime. No one believed him, all journals rejected his articles, and their reviews were insulting. What "Hirsch number" would N.N. Semenov have had at the time when his experiments were considered erroneous and his theory of chain reactions was ignored? What "Hirsch number" would N.I. Lobachevsky have had, who was literally derided, and whose geometry was considered delusional?

Of course, all these people would have had an unenviable $h$-index. Anatole France is right: science is infallible, but the learned often err. New ideas are accepted with difficulty and unwillingly. Rather cunningly, the reason was noted by J. Keynes, the founder of the theory of convergence: "The difficulty lies not so much in developing new ideas as in escaping from old ones." Old ideas have deserved trust and have been proved and reliable, while the creators of new ideas have to wait and hope, following Marina Tsvetaeva:

My poems, like precious wine,

Will have their time.

New ideas do have their time .... Often after their creators have gone to Eternity.

The Hirsch index is high when you are a success in recognized, sustainably fruitful, and popular scientific areas in which many people work and which have developed research teams and stable horizontal connections between them. However, if you are developing new, hardly known, out-of-main-stream areas, do not expect a good "Hirsch number." Yet this is a bad sign not for you but rather for it because it does not stimulate the generation and development of new ideas and areas. It does not encourage one to move into the future; it measures only the present and the past. This is its weakness.

If you are not too greedy for fame and do not bother with the Hirsch index, do not hesitate to undertake new areas, turning the unpredictable into the inevitable. This is the meaning of the charming game called science. The Hirsch index has nothing to do with it.... Science is the wrong place for it.

Academician A.L. Buchachenko, Institute of Problems of Chemical Physics, Russian Academy of Sciences e-mail:abuchach@chph.ras.ru Translated by B. Alekseev 